\title{
END PRODUCTS OF COMETARY EVOLUTION: COMETARY ORIGIN OF EARTH-CROSSING BODIES OF ASTEROIDAL APPEARANCE
}

\author{
G.W. WETHERILL \\ Department of Terrestrial Magnetism \\ Carnegie Institution of Washington \\ 5241 Broad Branch Road, N.W., \\ Washington, D.C. 20015
}

\begin{abstract}
Because there is no necessary connection between the time required to remove the volatile component of a cometary nucleus by solar heating (physical lifetime) and the dynamical lifetime of a comet, it is possible that a comet may evolve into an observable object of asteroidal appearance. Almost all comets have dynamical lifetimes much shorter than their physical lifetimes and in these cases complete loss of volatiles will not occur. Mechanisms do exist, however, whereby a small but significant fraction of comets will have longer dynamical lifetimes, permitting them to evolve first into Jupiter-family short period comets and then into comets with relatively safe decoupled orbits interior to Jupiter's orbit. Observed Jupiter-family objects of asteroidal appearance (e.g., 1983SA) are much more likely to be of cometary rather than asteroidal origin. "Decoupling" is facilitated by several mechanisms: perturbations by the terrestrial planets, perturbations by Jupiter and the other giant planets (including resonant perturbations) and non-gravitational orbital changes caused by the loss of gas and dust from the comet. The dynamical time scale for decoupling is probably $10^{5}-10^{6}$ years and almost all decoupled comets are likely to be of asteroidal appearance. Once decoupled, the orbits of the resulting Apollo-Amor objects will evolve on a longer $\left(10^{7}-10^{8}\right.$ year) time scale, and the orbital evidence for these objects having originally been comets rather than asteroids will nearly disappear. Statistically, however, a large fraction of the bodies in deep Earth-crossing orbits with semi-major axes $\gtrsim 2.2 \mathrm{AU}$ are likely to be cometary objects in orbits that have not yet diffused into the steady state distribution. For plausible values of the relevant parameters, estimates can be made of the number of cometary Apollo-Amor "asteroids," the observed number of Earthcrossing active and inactive short period comets, and the production rate of short period comets. These estimates are compatible with other theoretical and observational inferences that suggest the presence of a significant population of Apollo objects that formerly were active comets.
\end{abstract}

\section{Introduction}

About 50 bodies of asteroidal appearance brighter than absolute magnitude $\mathrm{V}(1,0)=$ $18(1.05 \mathrm{~km}$ diameter for visual geometric albedo 0.10$)$ have been discovered in orbits with perihelia interior to Earth's aphelion. These are commonly termed "Apollo objects," named after the first object of this lind to be discovered, 1832 Apollo. A similar number 
of bodies (Amor objects) have been discovered in somewhat larger orbits (perihelia 1.02 to $1.3 \mathrm{AU}$ ). Dynamical studies (Wetherill and Williams, 1968) have shown that as a result of secular perturbations and close encounters to planets, the orbits of Amor objects often become Earth-crossing, and similarly, Apollo objects often become Amor objects. For this reason, these two populations are not fundamentally distinct from one another and can be considered as a single population of Earth-approaching Apollo-Amor objects. Only a small fraction of the total number of these bodies has been discovered. The total number of Apollos brighter than $V(1,0)=18$ has been estimated to be $800 \pm 300$, and the number of Amors to be $1500 \pm 500$ (Shoemaker, 1977), whereas only about 100 of these bodies have been discovered so far.

The mean dynamical lifetime of Earth-crossing bodies $\left(10^{7}-10^{8}\right.$ years $)$ is short in comparison to the age of the Solar System, and a source is required to maintain the observed quasi-steady state number of bodies. The most plausible source regions are those parts of the Solar System known to contain a large number of bodies in the size range of ApolloAmor objects in relatively stable orbits: the main asteroid belt and the sources of comets in the trans-Neptunian Solar System.

Öpik $(1961,1963)$ was the first to quantitatively consider the nature of the source. He concluded, assuming only dynamical mechanisms known at the time, that it was not possible to transfer enough material from the asteroid belt into Earth-crossing orbits to maintain the observed number of bodies in a steady state. He therefore proposed that these objects are the devolatilized nuclei of short period comets. Their orbits differ from those of most Earth-crossing short period comets in that in almost all cases their aphelia are well inside $(<4.3 \mathrm{AU})$ the orbit of Jupiter. The existence of one active comet, $\mathrm{P} /$ Encke, in such an orbit showed that evolution of a comet into a "decoupled" orbit of this kind must be possible.

Subsequently, dynamical mechanisms capable of supplying approximately the observed steady state number of Apollo-Amor objects from the asteroid belt were discovered (Williams, 1973; Wetherill and Williams, 1979; Wisdom, 1983, 1985; Wetherill, 1988). Although these advances eliminated the crucial need for a cometary source, they do not in any way argue against a contribution of comparable magnitude from inactive comets. In fact, a body of circumstantial evidence has been accumulating indicating that comets exhibit a wide range of cometary activity, associated with the fraction of their surface that is active, and that at least many comets evolve into dormant or extinct states characterized by apparent absence of cometary activity (reviewed by Weissman et al., 1989). It seems likely that old comets never die, they just fade away.

This article reviews the present state of understanding of the dynamic mechanisms whereby the orbits of some comets evolve into those observed for Apollo-Amor objects. It will be concluded that appropriate mechanisms exist, and their characteristic time scale is one or two orders of magnitude longer than the active physical lifetime of most comets. Therefore it may be expected that most of those comets that do undergo this orbital evolution will no longer exhibit cometary activity, except insofar as they are "rejuvenated" following cratering collisions, or eruptions caused by buildup of internal gas pressure. 


\section{Stages in the Orbital Evolution of Short Period Comets}

Almost all observed active comets have perihelia inside the orbit of Jupiter, and aphelia beyond Jupiter. In order to supply a significant number of bodies in Apollo-Amor orbits, it is necessary that a small but sufficient fraction of cometary aphelia evolve inward to values more than about 0.6 AU inside Jupiter's perihelion. Several mechanisms exist that are likely to contribute toward this necessary decoupling:

(1) Close encounters to Earth and Venus, leading to random changes in the aphelion distance, the accumulation of which will occasionally lead to decoupling.

(2) Non-gravitational orbital changes that are the dynamical reaction to the momentum loss accompanying emission of gas and dust from an active comet.

(3) Multi-body perturbation of cometary orbits by the giant planets alone, including the effects of commensurability resonances.

These processes are by no means exclusive of one another. More likely, they will interact in a non-linear manner, and their combined effect may therefore be greater than that of any one alone. Even very rough estimates of the effect of these mechanisms lead to the conclusion that it is extremely unlikely that decoupling directly from a long-period cometary orbit is possible. Therefore attention should be centered on short-period comets, and in particular, Jupiter-family comets (defined here as those with aphelion less than 9 $\mathrm{AU}$ ), for which relatively little change in aphelion is necessary to achieve decoupling.

Taking the foregoing into consideration, the orbital evolution of Apollo-Amor objects derived from cometary sources can be divided into three stages:

(1) Evolution of the original trans-Neptunian orbit into a Jupiter-family orbit.

(2) Decoupling of the Jupiter-family orbit from Jupiter.

(3) Evolution of the decoupled Apollo-Amor orbit on a $10^{7}-10^{8}$ yr. time scale, terminated by transfer back into Jupiter-crossing, or by collision with a terrestrial planet.

In reality, of course, this three-stage orbital evolution is a continuous process, and the theoretical studies that have been made of it are equivalent to treatment of it as such a process. For this reason, this division of the evolution into three stages should not be thought of as a "scenario" but rather as a conceptually convenient description of the complete and continuous orbital evolution.

\section{The First Stage: Evolution of Trans-Neptunian Comets Into Jupiter-Family Short Period Comets.}

The fundamental requirement for decoupling a comet into an Apollo-Amor orbit is reduction of its aphelion from its initial trans-Neptunian value to that of a Jupiter-family comet and, ultimately into a body with aphelion safely (for $10^{7}-10^{8}$ years) inside the orbit of Jupiter.

The process begins with perturbation of the distant cometary orbit, reduction of its perihelion into a Jupiter-crossing orbit, followed by orientation of its velocity vector relative to that of Jupiter in such a way that its aphelion lies not too far beyond Jupiter, and its perihelion well within Jupiter's orbit. Everhart $(1972,1977)$ and Stagg and Bailey (1989) have studied the capture of long period comets into short period orbits by this process, and concluded that although capture following a single event is improbable, an adequate number of captures can occur as a result of the accumulation of a large number of perturbations by the giant planets. 
Problems with the orbital distribution of short period comets have been recognized for some time (Havnes, 1967). Recent work by Duncan et al. (1988), using a straightforward numerical integration approach, shows that the calculated distribution of inclinations resulting from capture of long period comets is very different from the observed distribution of inclinations of short period and Jupiter-family comets. Eighty-three percent of the observed short period comets with aphelia less than $9 \mathrm{AU}$ have inclinations less than $20^{\circ}$, and with one exception (60\%) the maximum observed inclination of these Jupiter-family comets is $45^{\circ}$. For short period comets as a whole $68 \%$ have inclination $<20^{\circ}$. In contrast, $82 \%$ of the calculated short period cometary orbits captured from originally parabolic orbits with an isotropic distribution of inclinations have inclinations greater than $37^{\circ}$. These authors conclude that prior to capture into a Jupiter-crossing orbit these comets must have had an inclination distribution dominated by low inclinations (e.g., $i \leqslant 18^{\circ}$ ). They illustrate this conclusion with results for a distribution of this kind for the case of short period comets derived from a "Kuiper belt" of comets with initial aphelia of $50 \mathrm{AU}$ and initial perihelia between 20 and $30 \mathrm{AU}$ (Fernández, 1980). Stagg and Bailey (1989) point out that the results of Duncan et al. for parabolic comets are statistically limited. Although capture was defined to include comets with perihelia as large as $2.5 \mathrm{AU}$, only 12 cases were found with periods less than 200 years. Good statistics are obtained for the Kuiper belt case, but they point out that these results may be questioned because of the enhanced giant planet masses $(40 \mathrm{x})$ that had to be used to obtain these good statistics.

The author has carried out Monte Carlo calculations of the capture of comets into short period orbits using the technique of Arnold (1965) based on the equations of Öpik (1951). Shoemaker and Wolfe (1984), and Fernández and Ip (1983) have also applied this technique to problems of cometary orbital evolution. Serious questions should be raised concerning the validity of this technique for the study of such problems, because the time scale for close approaches to planets, particularly Jupiter, is short compared with the precession period of the argument of the comet's perihelion, violating an assumption made in the derivation of Öpik's equations.

Nevertheless, it was found that use of this technique reproduced quite well the conclusions reported by Duncan et al. (1988) for the two cases they studied. The results of only a moderate extension of their initial conditions using this technique are shown in Figure 1. This is the calculated distribution of final inclinations of 472 Jupiter-captured Jupiter-family comets (aphelion <9 AU) at the time they reached perihelion in the visible range $(<1.5 \mathrm{AU})$. The evolution of $2 \times 10^{5}$ initial near-parabolic orbits (aphelion $=3$ $\times 10^{5} \mathrm{AU}$ ) was calculated, assuming Jupiter-crossing perihelia between 4 and $6 \mathrm{AU}$, a uniform distribution in $\cos i$ for inclinations between 0 and $180^{\circ}$, and no enhancement of masses. In agreement with the results of Duncan et al., the final inclination distribution is very different from that observed for Jupiter-family comets. Fifty-five percent of the final orbits have inclinations $>37^{\circ}$. Sixteen percent of the bodies are in retrograde orbits, whereas none of the 108 observed Jupiter-family comets in Marsden (1986) are in retrograde orbits.

This discrepancy is only apparently alleviated by specification of long period comets with initial perihelia between 20 and $30 \mathrm{AU}$, instead of Jupiter-crossing (Figure 2). Although the inclination distribution is more similar to that observed, the efficiency for capture of these bodies into Jupiter-family orbits is found to be 1000 times smaller. It is difficult to 


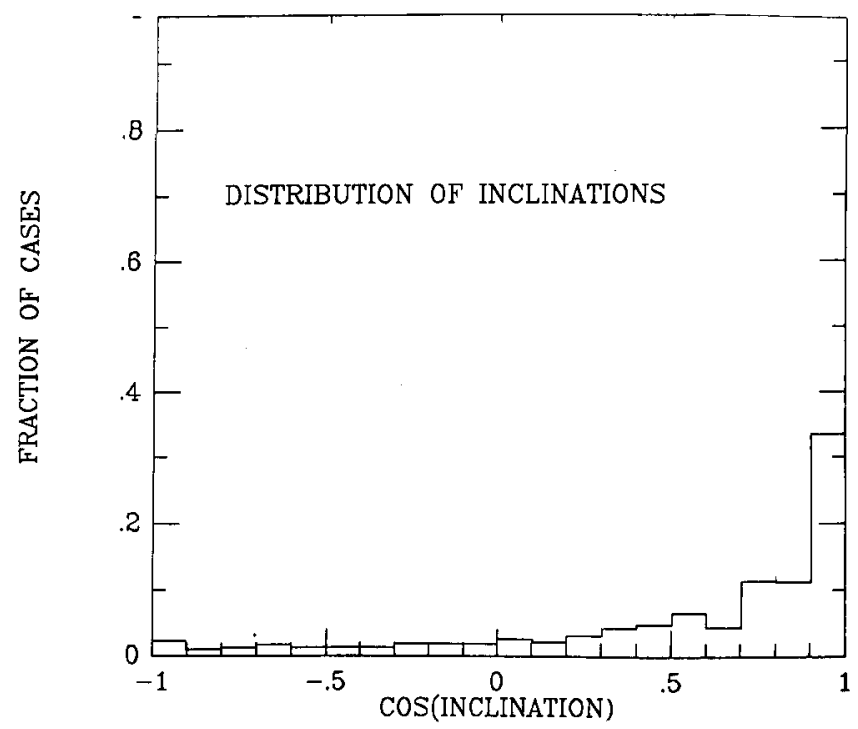

Figure 1. Calculated final distribution of inclinations of 472 Jupiter-family comets (aphelia $<9 \mathrm{AU}$, perihelia $<1.5 \mathrm{AU}$ ) captured from $2 \times 10^{5}$ bodies initially in nearparabolic orbits (aphelia $=3 \times 10^{5} \mathrm{AU}$ ). The initial perihelia were randomly distributed between 4 and $6 \mathrm{AU}$, and the initial inclinations were isotropically distributed.

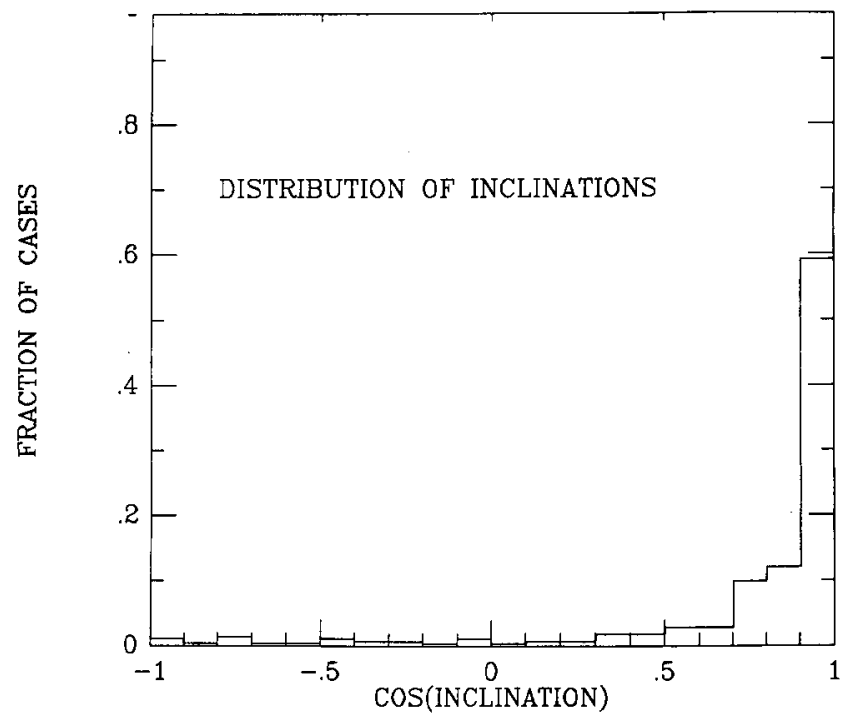

Figure 2. Calculated final distribution of inclinations of 334 Jupiter family comets captured from $10^{8}$ initial orbits the same as those of Figure 1, except that the initial perihelia were randomly distributed between 20 and $30 \mathrm{AU}$. 
understand how the distribution of initial perihelia could be sufficiently concentrated toward large values to allow the contribution of the bodies with larger perihelia to so dominate the production of Jupiter-family comets. Extension of these calculations to a wide range of initial orbital distributions, including aphelia of 200,2000 , and $10^{4} \mathrm{AU}$ leads to the same conclusion reached by Duncan et al. (1988): agreement with the observed orbital distribution of short period comets seems to require a source with an initial inclination distribution similar to the low inclination distribution observed. The results of a Monte Carlo calculation with no enhancement of masses for a source identical to that of Duncan et al. (1988) (aphelia=50 AU) are shown in Figure 3. When combined with the earlier work of Duncan et al. (1987), this implies a source with aphelion $\lesssim 2000 \mathrm{AU}$, because more distant bodies in the cometary zone may be expected to have had their inclination randomized by passing stars. This conclusion may be difficult to reconcile with estimates of the magnitude and expected number of comet showers from a massive "inner Oort cloud" at greater distances (Hills, 1981; Weissman, 1986). One would expect the inclination distribution resulting from capture of shower comets isotropically distributed into short period orbits to contain many more high inclination orbits. Consideration of this question lies beyond the scope of this review.

The mechanisms by which comets in the non-isotropic inner portion of the cometary region may be perturbed into Neptune-crossing are uncertain at present. Fernández (1980) proposed that this was accomplished by perturbations caused by an unobserved population of Ceres-size bodies beyond the orbit of Neptune. More recently, Torbett (1989) has

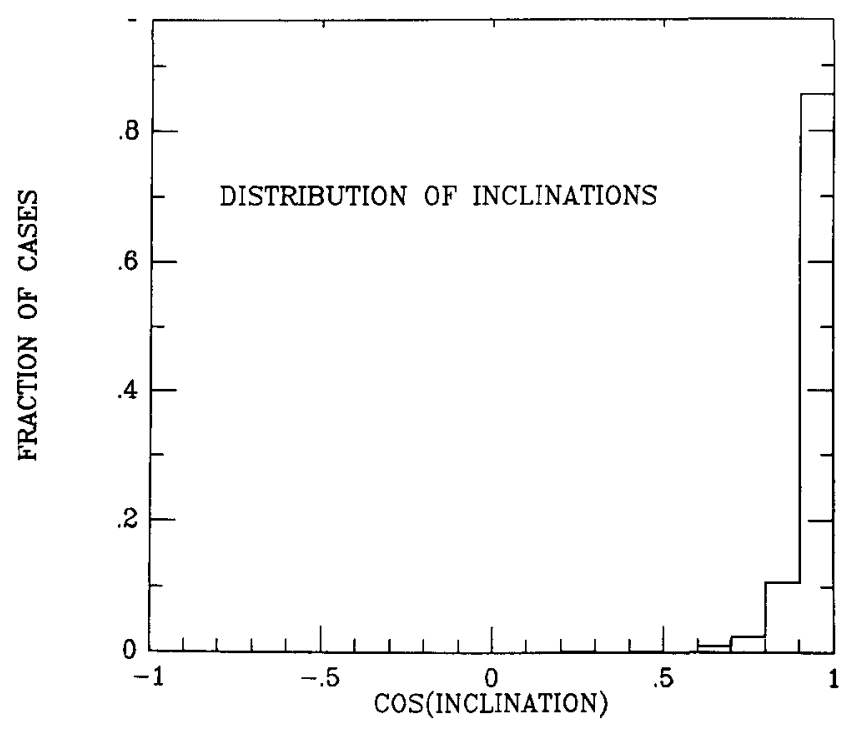

Figure 3. Final inclination distribution of 783 Jupiter-family comets calculated from 2500 initial orbits with aphelia of $50 \mathrm{AU}$ and perihelia between 20 and $30 \mathrm{AU}$. Initial inclinations were randomly distributed in $\cos i$ for inclinations between $0^{\circ}$ and $18^{\circ}$. Planetary masses were not enhanced. This calculated distribution is similar to the observed distribution. 
reported numerical calculations showing that perturbations by the major planets produce chaotic zones in the nearby trans-Neptunian region, out to $\sim 100 \mathrm{AU}$ or more, that may cause comets in this region to evolve into Neptune-crossing orbits, provided their initial orbits are sufficiently eccentric. These two mechanisms require a source region just beyond Neptune's orbit. It is also possible that comets with semi-major axes $\sim 2000 \mathrm{AU}$ may be perturbed into Neptune-crossing from the outermost-boundary of the non-isotropic region as a result of perturbations by the few most deeply penetrating passing stars. Such a source would exhibit major stochastic fluctuations in magnitude. Those comets perturbed only to Neptune-crossing would require $10^{8}-10^{9}$ years to achieve perihelia less than 1.5 AU and become visible. Conceivably, an event of this kind could be related to the apparent factor of $\sim 2$ increase in cratering rate at $1 \mathrm{AU}$ during the last $\sim 500$ million years (Shoemaker et al., 1979; Grieve and Dence, 1979; Weissman, 1989; Wetherill, 1989).

\section{The Second Stage: Decoupling of Jupiter-Family Comets From Jupiter}

The relatively high observed steady-state abundance of Apollo-Amor objects is the result of the fairly long ( $\sim 10^{7}$ to $10^{8}$ year) lifetime of Earth-approaching bodies that avoid close approaches to Jupiter because their aphelia are well within its orbit. Compared with the large perturbations caused by close encounters with Jupiter, the perturbations caused by close approaches to the terrestrial planets and temporary residence in Joviancommensurability chaotic zones are small and/or infrequent, leading to these relatively long steady-state lifetimes. As mentioned earlier, transfer of a Jupiter-family orbit into a "protected" orbit of this kind can be facilitated in several known ways: by close encounters to the terrestrial planets, by non-gravitational acceleration associated with mass loss from active comets, and by the gravitational influence of Jupiter.

A Jupiter-family comet usually develops an easily observed coma when its perihelion evolves inside about 1.5 AU. The same Monte Carlo calculations discussed in the previous section show that about $65 \%$ of the Jupiter-family comets with perihelia $<1.5$ evolve further to become Earth-crossing while still in Jupiter-crossing orbits. The remainder are lost before their perihelion becomes Earth-crossing. When the comet is Earth-crossing, close approaches to Earth become possible, and in principle, a sufficiently strong perturbation by Earth could decouple the aphelion from Jupiter before Jupiter itself perturbs the comet into a hyperbolic Solar System escape orbit. This tug-of-war between Earth (or Venus) and Jupiter is an unfair contest. Jupiter's perturbations are so much more frequent and strong that Jupiter almost always wins. On the other hand, quantitative studies of the orbital evolution of decoupled comets shows that Earth need not win very often. A quite low production rate of these objects, one body with absolute nuclear magnitude brighter than $\mathrm{V}(1,0)=18$ in $5.7 \times 10^{4}$ years, is sufficient to provide half the observed Apollos (Wetherill, 1988). The problem is that of making a usefully quantitative estimate of how infrequently Jupiter loses.

Relatively little attention has been given to this difficult but important question. The difficulty stems from the long time scale and the low probability of decoupling. Numerical integration could provide useful results only if enough long $\left(10^{5}-10^{6}\right.$ year $)$ integrations were carried out to identify a small, but reasonable, number (e.g., five) of decoupling events. Almost all of these lengthy integrations would come to naught: the effect of even 
an unusually promising "run" of terrestrial planet encounters would often be vitiated by a single perturbation by Jupiter. Calculations of this kind have not yet been attempted.

Some relevant results have been obtained by use of the Öpik-Arnold Monte Carlo approach to long-term orbital evolution. In this way Wetherill (1968a) estimated that about 1 in 500 Jupiter-family comets would be decoupled. Reservations were expressed by the author about the reliability of this result because of an unphysical aspect of the way in which Jupiter perturbations were calculated. Jupiter's influence was included only for orbits that actually crossed the orbit of Jupiter, and the overwhelmingly dominant effect of Jupiter suddenly fell to zero as soon as a terrestrial planet perturbation succeeded in perturbing the comet's aphelion inside Jupiter's perihelion. Subsequently, the most gross aspect of this situation was eliminated by modification of the calculation of Jupiter perturbations to gradually introduce the effect of Jupiter encounters beyond 4.30 AU (Wetherill, 1979). Although this treatment remained quite crude, it did reproduce the results of an investigation of the stability of Jupiter-approaching orbits using numerical integration by Lecar and Franklin (1973). This modification has been used in subsequent studies of the orbital evolution of bodies in the inner Solar System and has been considered satisfactory primarily because the results of these studies were found to be very insensitive to the exact way in which these distant Jupiter perturbations were introduced. In fact, no major difference was found when they were totally ignored.

This is not the case for the decoupling problem. Here we are concerned with the few cases in which Earth wins the contest, rather than the much more common one in which Earth loses. The outcome will certainly be dependent on the manner in which Jupiter perturbations are introduced. In addition, as mentioned in the previous section, one must question the validity of this Monte Carlo technique when the time scale between Jupiter encounters is short compared with the period of precession of the argument of perihelion. Nevertheless, it is thought that in view of the agreement that was obtained with the results of Duncan et al. (1988), use of this technique to illustrate some aspects of the decoupling problem could be of interest.

An initial assemblage of Neptune-crossing comets with aphelion equal to $50 \mathrm{AU}$ were used to generate 294 Jupiter-family orbits with perihelia randomly distributed between 20 and $30 \mathrm{AU}$, and $\cos i$ randomly distributed for inclinations between $0^{\circ}$ and $18^{\circ}$. This corresponds to the short period comet source of Duncan et al. (1988) discussed in the previous section. The calculation of the evolution was stopped when the perihelion became less than $1 \mathrm{AU}$, when the comet was ejected from the Solar System, or when the comet struck a planet. All of the aphelia were still Jupiter-crossing at that time.

The orbits with perihelia less than $1 \mathrm{AU}$ and inclination less than $18^{\circ}$ were then selected as starting orbits for further Öpik-Arnold Monte Carlo calculations. The restriction to low inclination was made primarily for a practical reason. At high inclinations the free oscillations of the secular perturbations in eccentricity become large. The effect of these oscillations has been included in some Monte Carlo calculations (Wetherill 1968b) and it was found that the oscillations had only a small effect on the outcome of the calculations because of the correlation between eccentricity, inclination, and argument of perihelion. This tends to limit the effect of the potentially important large excursions in eccentricity because they occur when $\omega$ is near $90^{\circ}$ or $270^{\circ}$, which is when the perihelion and aphelion are well above or below the plane of the Solar System and marginally Earthcrossing orbits will not actually intersect Earth's orbit. The secular resonances mapped 
by Williams and Faulkner (1981) were found to be of much greater importance, particularly the " $\nu_{6}$ " resonance that lies near $2.04 \mathrm{AU}$ for inclinations $<15^{\circ}$. Inclusion of free oscillations leads, however, to serious complications in the calculations when the effects of the $\nu_{6}$ resonance are also included. For this reason, the Monte Carlo programs currently in use do not include the free oscillations. Only two observed Earth-crossing Jupiter-family comets ( $\mathrm{P} /$ Brorsen) have inclinations well above $18^{\circ}$, and this restriction is not severe for calculations made for illustrative purposes.

The first case studied was the simplest one, in which no resonance effects were included. Four hundred Monte Carlo runs of each of the 294 Jupiter-family comets were made, resulting in 95 cases of comets decoupled into orbits with aphelia $<4.35 \mathrm{AU}$, corresponding to a decoupling efficiency of $0.81 \times 10^{-3}$. This is in agreement with the suggestion that the value of $2 \times 10^{-3}$ reported in Wetherill (1968a) was somewhat too high. The distribution of eccentricities and semi-major axes at the time of decoupling is shown in Figure 4.

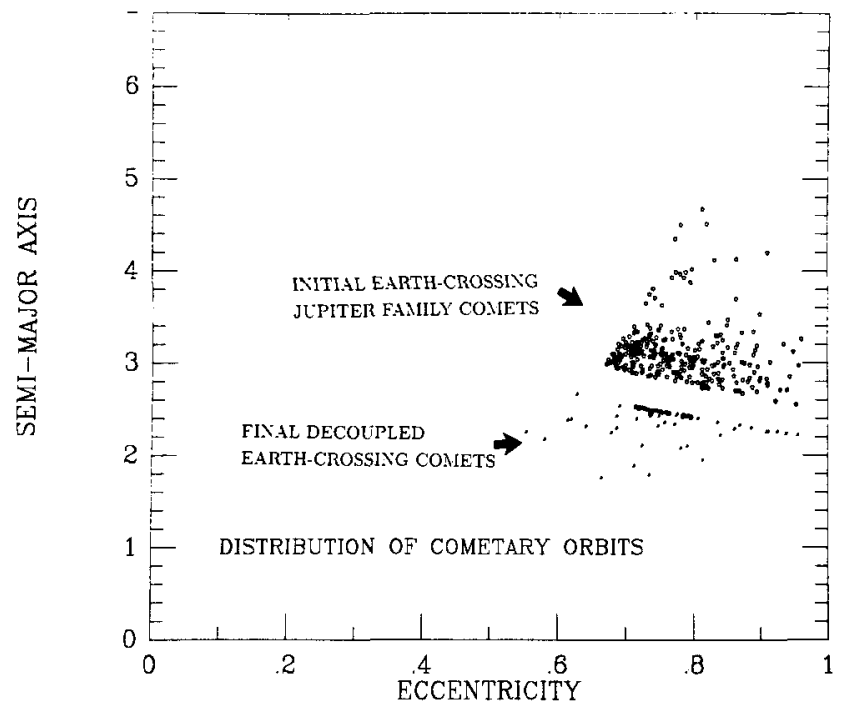

Figure 4. Calculated distribution of initial and final eccentricities and semi-major axes for decoupled comets. The upper group of open points are the initial Jupiter-family orbits. These are bounded on the left by the requirement that their perihelia be less than $1 \mathrm{AU}$, and bounded below by the requirement that their aphelia be Jupiter-crossing. The lower group of solid points are the decoupled comets resulting from the Monte Carlo calculations. These are bounded on the left by the requirement that the perihelion be less than $1 \mathrm{AU}$, and bounded above by the requirement that the aphelion be less than $4.35 \mathrm{AU}$.

Examination of the detailed orbital evolution of these decoupled comets showed that decoupling occurs gradually as a result of a series of Earth and Venus encounters. From time to time the aphelion of the comet is perturbed a bit inside Jupiter's perihelion, e.g., to $4.85 \mathrm{AU}$. This decreases the frequency of Jupiter encounters somewhat, providing an opportunity for terrestrial planet perturbations to reduce the aphelion somewhat more. 
Quite often, the opposite effect occurs, and the aphelion is perturbed back into Jupitercrossing. Rarely, but significantly, a chance series of several terrestrial planet encounters in sequence will reduce the aphelion to the point where the competition between Earth and Jupiter becomes less unfair, and further evolution into a decoupled orbit becomes relatively probable.

As stated earlier, there are several other processes that are likely to facilitate decoupling. One of these is a consequence of the enhanced long range perturbations by Jupiter when the comet's semi-major axis is close to a Jovian commensurability resonance. On the long ( $>10^{6}$ year) time scale for evolution of bodies in the terrestrial planet region, these resonances, particularly the $3: 1$ resonance at $2.5 \mathrm{AU}$, are of great importance because of their associated chaotic zones (Wisdom, 1983) and the large excursions in eccentricity experienced by bodies that remain in these resonances for $\sim 10^{6}$ years. In the present problem, however, the semi-major axes of the comets are "scanned" over these resonances by Jupiter encounters on a much shorter time scale. For the same reason, the effects of the secular resonances, particularly $\nu_{6}$, also are of reduced importance for Jupiter-crossing bodies. On a $10^{4}$ year time scale, however, average $\nu_{6}$ amplitudes of about \pm .075 in eccentricities may be expected, and these have been found to be important in "smearing" the definition of Earth-crossing by permitting Earth-crossing to be re-established relatively easily after a Jupiter perturbation increases the comet's perihelion only slightly beyond Earth's orbit. The principal effect of the Jovian commensurability resonances for Jupitercrossing bodies is the temporary $\left(10^{4}\right.$ to $\left.10^{5} \mathrm{yr}\right)$ protection from close Jupiter encounters provided by librations while in these resonances, as illustrated by numerical integrations by Milani et al. (1989). While the comet is protected in this way, Earth and Venus have a greater opportunity to decrease the comet's aphelion.

There is insufficient information at present to model these effects very quantitatively. The published results of Milani et al., although not statistical, suggest that while the comet is in these resonances, the frequency of close Jupiter encounters may be reduced by a factor of 10 , and possibly by a factor of 100 . The effect of this protection has been explored by simply assuming that the encounter probability with Jupiter is decreased by a given factor when the semi-major axis of the comet is within $\pm .02 \mathrm{AU}$ of the $4: 1,3: 1$, $5: 2,7: 3$, or $2: 1$ resonances. A maximum $\nu_{6}$ eccentricity amplitude of .075 was permitted for bodies in the vicinity of the $\nu_{6}$ resonance. Assuming a resonance protection factor of 10, 300 Monte Carlo calculations of each of the same 294 Jupiter-family comets were carried out. Two hundred eighty nine decoupled comets were found, corresponding to a decoupling efficiency of $3.3 \times 10^{-3}$. (A larger value of decoupling efficiency, $1.1 \times 10^{-2}$ was found for the possibly excessive protection factor of 100.) The semi-major axis and eccentricity distribution of the resulting decoupled comets is shown in Figure 5, and their perihclion distribution in Figure 6. The semi-major axes tend to be clustered between 2.1 and $2.5 \mathrm{AU}$. Perihelia cover a wide range, but are more concentrated between 0.5 and $0.9 \mathrm{AU}$. The perihelion distribution is distinctly different from the strong concentration of initial Earth-crossing perihelia near $1 \mathrm{AU}$ found for meteorites and Apollo objects of asteroidal origin (Wetherill, 1985, 1987, 1988).

The times at which decoupling occurred are shown in Figure 7. These are distributed, but most comets are decoupled after $10^{5}$ to $10^{6}$ years of evolution from their initial Jupiterfamily orbit. These times are longer than estimates of the physical lifetime of comets of $\sim 7 \times 10^{3}$ years for typical Jupiter-family periods (Weissman, 1980). For this reason it 


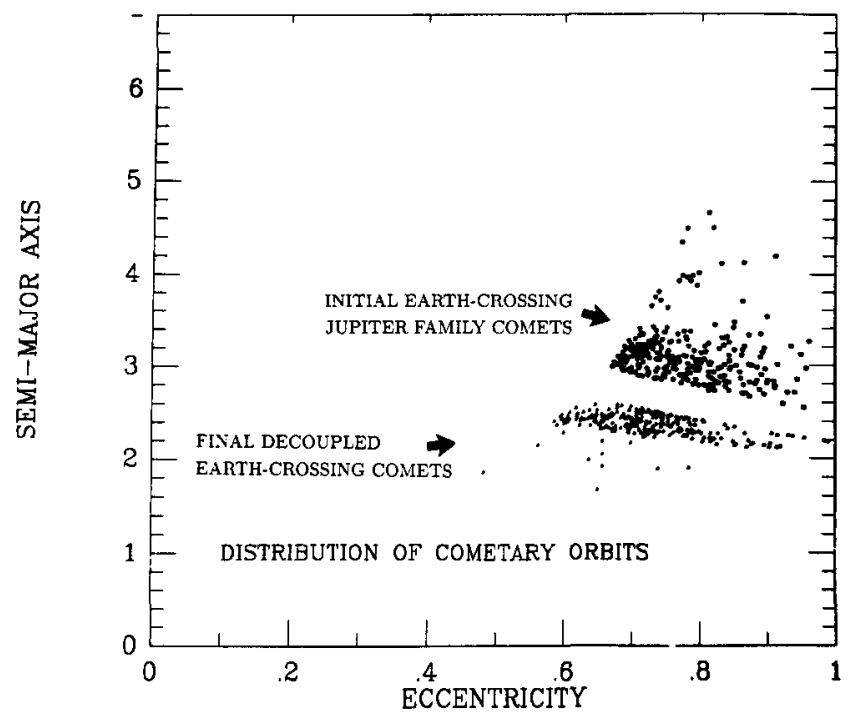

Figure 5. Same as Figure 4, except that the effects of commensurability and secular resonances have been introduced, as described in the text.

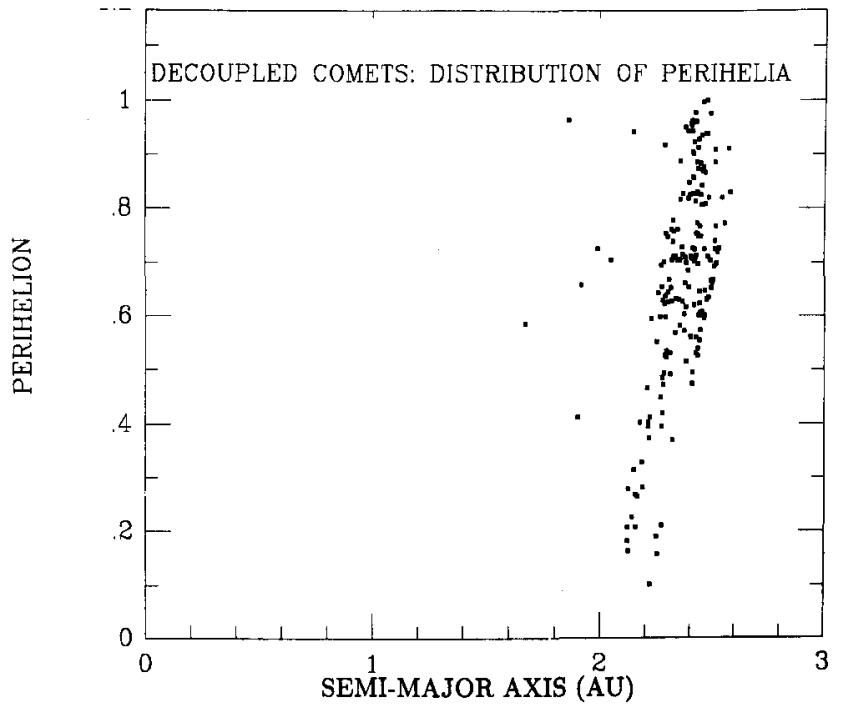

Figure 6. Initial distribution of perihelia and semi-major axis of decoupled comets. The initial rather restricted distribution evolves into the steady-state distribution shown in Figure 8. 
may be expected that almost all decoupled comets will be extinct or dormant, and will be of asteroidal appearance. In a few cases, totalling $\sim 0.5 \%$ of the decoupled comets, decoupling times in the $10^{3}-10^{4}$ year range are found, and therefore the appearance of one presently active comet (Encke) out of the possible several hundred cometary Apollo objects is not unexpected.

The case illustrated by Figures 5, 6, and 7 can be used to demonstrate some quantitative relationships between the decoupling efficiency and the capture rate into Jupiter-family orbits, the steady state number of Apollo objects, and the number of active and inactive Jupiter-family comets in Earth-crossing orbits.

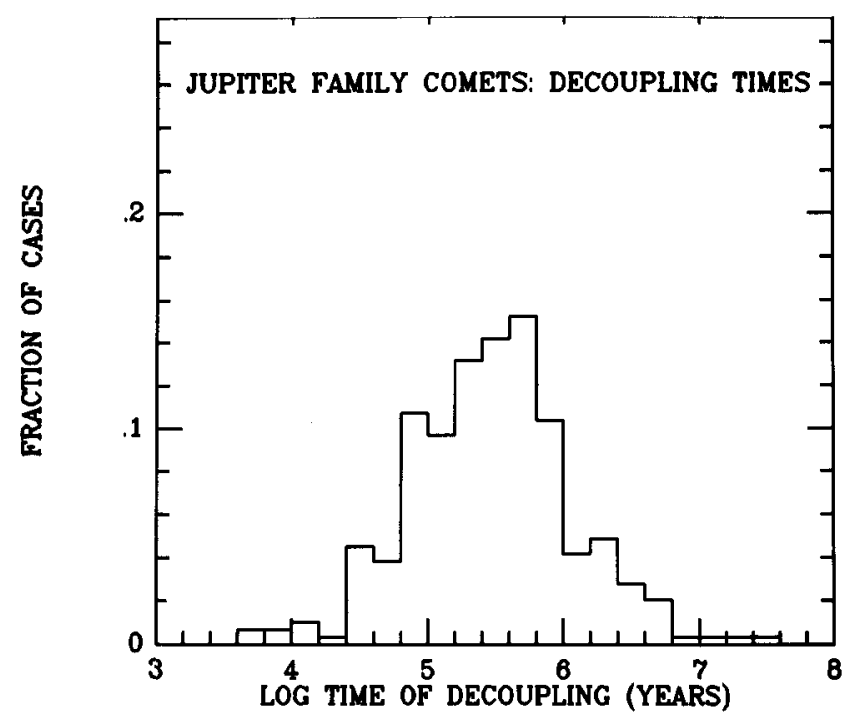

Figure 7. Calculated distribution of elapsed times at which Jupiter family comets became decoupled from Jupiter (aphelion <4.35 AU). Typical decoupling times are in the general range of $10^{4}-10^{6}$ years, but many lie outside this range. Except for the few bodies with very short decoupling times, most of these bodies may be expected to have an asteroidal appearance.

The nominal benchmark to be used for scaling these comparisons is based on the conclusion reached in Wetherill (1988), that an injection rate $r_{A}$ of one decoupled comet with absolute nuclear magnitude brighter than $\mathrm{V}(1,0)=18$ per $5.7 \times 10^{4}$ years will provide a steady-state number of Apollo objects equal to that calculated from asteroidal sources. For a geometric visual albedo $\mathrm{p}_{v}=0.04$, this corresponds to a diameter of $1.66 \mathrm{~km}$.

This nominal requirement $r_{\mathrm{A}}=1.75 \times 10^{-5} \mathrm{yr}^{-1}$ is related to the production rate of Earth-crossing Jupiter-family comets $\left(r_{c}\right)$ and the decoupling efficiency $f_{D}=3.3 \times 10^{-3}$ by

$$
r_{c}=r_{\mathrm{A}} / f_{\mathrm{D}}=5.3 \times 10^{-3} y r^{-1}
$$


Here $r_{c}$ is defined to include only Earth-crossing Jupiter-family comets with aphelia less than $9 \mathrm{AU}$ and with nuclear magnitude brighter than 18, after any mass loss incurred during decoupling. This is similar to the commonly used estimate of one short period comet per century (Fernández, 1985). The Monte Carlo calculations directly provide the steady-state number of decoupled comets, by use of the procedure described earlier, with an appropriately smaller $\left(10^{3}\right.$ years) sampling interval (Wetherill, 1979). For a Monte Carlo injection rate of 1 Jupiter-family comet/year, the calculation yields a steady state number of $3.2 \times 10^{4}$ Earth-crossing Jupiter-family comets of the required brightness. For the production $r_{c}$, from equation (1), the nominal number of steady-state comets will be equal to $r_{c}$ times $3.2 \times 10^{4}$, i.e., 170 . This number includes both active and inactive comets. The Monte Carlo calculations also provide the steady-state number of active comets, i.e., those comets less than 7000 years old, with "age" being defined as the time since the perihelion of the Jupiter-family comet first crossed $1 \mathrm{AU}$. This steady-state number is found to equal 7.7 active decoupled comets with nuclear magnitude brighter than $\mathrm{V}(1,0)$ $=18$. Nine active Jupiter-family comets in Earth-crossing orbits are included in the compilation by Marsden (1986). Diameters have been measured for only one of these, Tempel $2(11.2 \mathrm{~km})$, much larger than $1.66 \mathrm{~km}$ (Campins et al., 1988). Weissman (1989) estimates that there are 100 active Earth-crossing comets larger than $0.52 \mathrm{~km}$ diameter. Assuming that comet diameters are distributed in accordance with a cumulative power law index of -2 , and an albedo of 0.04 , Weissman's estimate corresponds to 9.8 active comets brighter than absolute magnitude 18 .

Thus, there is no obvious disagreement between observations of active Jupiter-family comets, the decoupling efficiency used, and the nominal assumption that half of the Apollo objects are comets of asteroidal appearance. The estimated number of inactive Jupiterfamily comets is considerably greater than the number of active Jupiter-family comets, and is not much less than estimates of the number of Apollo-Amor objects. Observed bodies of asteroidal appearance but with orbits similar to Jupiter-family comets (e.g., 1983SA) are more than 100 times as likely to be inactive Jupiter-family comets that have never been decoupled, as they are to be former Apollo-Amor objects with aphelia $\lesssim 4.3 \mathrm{AU}$. Even one Jupiter-crossing asteroidal Apollo-Amor with $\mathrm{V}(1,0)<18$ is unexpected, and these inactive Jupiter-family comets may provide the best opportunity to study cometary nuclei without interference from surrounding dust and gas.

In much of the literature on this subject, steady-state numbers $(N)$ are related to production rates $(p)$ and characteristic lifetimes $(\lambda)$ by the simple relationship

$$
N=p / \lambda
$$

It should be noted that in the present work steady-state estimates do not depend on any assumed mean lifetimes, nor upon use of this relationship. Calculations using this relationship are valid only for simple "reservoir" models of steady-state populations, which are not usually valid. As a consequence, steady-state numbers calculated in that way can lead to misleading conclusions. Monte Carlo calculations can provide entirely independent "lifetimes" whenever the meaning of the term lifetime is defined with sufficient precision to be calculated. For example, the "harmonic mean lifetime" (reciprocal of the mean sum of reciprocal lifetimes) is found to be $1.61 \times 10^{4}$ years for the migration of the perihelion of a Jupiter-family comet beyond Jupiter, and $4.72 \times 10^{4}$ years for the escape of the Jupiter-family comet from the Solar System. These "lifetimes" are more or less similar to the calculated mean elapsed time of $7.7 \times 10^{4}$ years in Earth-crossing orbit, calculated by 
summing the time intervals rather than their reciprocals. In contrast, the mean elapsed time before ejection from the Solar System is found to be much larger, $2.60 \times 10^{7}$ years. The reason for this difference is the occurrence of a long-lived "tail" in the elapsed time distributions caused by a relatively small number of bodies having long sojourns in purely Neptune-crossing orbits prior to ejection. These cases make a negligible contribution to the sum of reciprocal lifetimes, but dominate the lifetime when calculated as a simple sum. The "single reservoir" lifetime, calculated from the ratio of the steady-state number of Jupiter-family comets (170) to the nominal production rate of Jupiter-family comets from equation (1), is $3.2 \times 10^{4}$ years. Although much shorter than the very long mean elapsed time for escape, this quantity is in the general range of the harmonic mean lifetimes and the mean elapsed time in Earth-crossing orbit. It does not correspond exactly to any of these quantities, however, because the simple exponential decay associated with a single reservoir model is neither physically realistic, nor is it found by these calculations.

Another known process that can cause changes in the aphelion distance is the nongravitational reaction force associated with loss of momentum accompanying cometary mass loss (Whipple, 1950, 1951; Sekanina, 1969, 1971). In the first work on the source of Apollo objects, Öpik (1961) introduced the idea that this non-gravitational force was a possible mechanism by which Jupiter-family comets were decoupled from Jupiter to become Encke-like comets, and, following loss of their volatiles, to become Apollo objects.

Such non-gravitational accelerations have long been observed for comets (Marsden, 1985). On the average these effects are usually too small in magnitude to permit decoupling by this process alone during the lifetime of the comet. This situation is aggravated by the discovery that for the same comet, the sign of the non-gravitational force can change (Yeomans, 1988) over a period short in comparison with the active physical lifetime of the comet. As a consequence, the change in aphelion may vary with the square root of time as in random walk, rather than linearly with time. Under these circumstances, cumulative variations of aphelia even as large as $0.1 \mathrm{AU}$ may be rare. If these fluctuations in aphelion distance are indeed random in sign, they will be small in comparison with fluctuations caused by Jupiter perturbations, even those that occur during the active lifetime of the comet. For this reason, there is little basis for expecting that these non-gravitational forces play a major part in the decoupling of comets from Jupiter, but neither can a contribution by this effect be ruled out, particularly if the sign of the force remains the same long enough to provide a significant negative secular acceleration in semi-major axis, e.g., $\sim 0.2-0.3 \mathrm{AU}$. In such a case, this additional acceleration could hasten migration of the comet's aphelion into $\sim 4.8 \mathrm{AU}$, setting up the interplay of Jovian commensurability resonance and terrestrial planet perturbations found to eventually lead to decoupling.

As discussed earlier, commensurable resonance zones can be established by Jupiter perturbations, greatly reducing the frequency of close encounters to Jupiter. In combination with Earth or Venus close encounters, decoupling is facilitated. It is also known (e.g., $\mathrm{P} /$ Oterma) that all by itself Jupiter can transform a cometary orbit from one with perihelion beyond Jupiter to one with aphelion inside Jupiter's orbit. Observed changes of the latter kind often are associated with commensurability without libration, and involve repeated close encounters with Jupiter, with the comet's perihelion well beyond Earth's orbit. As a general rule, these orbital changes do not lead to permanent decoupling. It may be noted that Duncan et al. (1988) report cases with perihelia $<1.5 \mathrm{AU}$ for which the final aphelia were decoupled, even though terrestrial planet perturbations were not included in the calculations.

Because of the uncertainties described earlier in use of Monte Carlo calculations of 
this kind for Jupiter-crossing, they should be regarded as illustrative, rather than conclusive. The decoupling efficiencies found, in the range $10^{-2}$ to $10^{-3}$, permit relating various observed phenomena in an internally consistent way. Obtaining much more accurate decoupling efficiencies by numerical integration in the near future may be difficult, however. Perhaps the most promising approach would be a synergistic one of using the results of numerical integration to improve the Monte Carlo programs, and in return use the results of the Monte Carlo calculations to determine the most effective way to use available computational resources in investigations making use of numerical integration.

\section{The Third and Final Stage: Orbital Evolution of Decoupled Comets}

When a comet is in a near-Jupiter-crossing orbit, significant orbital changes occur on short time scales ( $~ 100$ years), usually leading to ejection from the Solar System in $10^{4}$ to $10^{5}$ years. After the comet's aphelion is less than the somewhat arbitrary value of $4.35 \mathrm{AU}$, the magnitude of perturbations by Jupiter and other major planets decreases considerably, except near commensurability and secular resonances. Furthermore, questions of the validity of the Monte Carlo calculations become less serious. Objects in decoupled orbits frequently evolve into orbits with smaller eccentricities and aphelia even when the effects of these resonances are included. A quasi-steady-state orbital distribution will evolve, primarily reflecting the effects of three factors: the initial orbital distribution, the tendency of perturbations by the terrestrial planets to randomize the orientation of the comet-planet relative velocity vector, and perturbation back into Jupiter-crossing, followed by nearly inevitable rapid ejection from the Solar System. The steady-state distribution will also be affected by the alternative ultimate fates of collision with a terrestrial planet or collisional destruction near aphelion in the asteroid belt, and the less quantifiable one of complete disintegration into a meteor stream. Because of the typical $10^{4}-10^{5}$ age at the time of decoupling, almost all decoupled comets would be expected to be of asteroidal appearance during this final $10^{7}-10^{8}$ period of their Solar System residence.

Steady-state orbital distributions of comets following decoupling have been calculated by use of the Öpik-Arnold technique (Wetherill, 1979, 1988). The result of a new calculation of this kind is given in Figure 8, using as initial orbits the decoupled comet orbits shown in Figure 6. This distribution may be compared with the calculated distribution of ApolloAmor objects of asteroidal origin. The latter distribution is the result of a new calculation, in which an improved procedure has been used for the calculation of the steady-state distribution. This eliminates the prominent band of objects near 2 A.U., in agreement with the earlier suggestion (Wetherill, 1988) that this feature may be an artifact. The remainder of the distribution is essentially unchanged.

Perhaps the most important result of comparison of Figure 8 and Figure 9 is the nearly complete overlap of the regions occupied by Apollo objects of different origin. Therefore it usually will be difficult to use the orbit of an Apollo object to support the case for an asteroidal vs. cometary origin. A vestigial record of the initial state is probably reflected by the relatively high population of deep Earth-crossing cometary Apollos with large semi-major axes and aphelia. Perhaps the higher inclination Apollo-Amor objects are also more likely to be of cometary origin. Scholl and Froeschlé (1986), however, report evidence that the $\nu_{16}$ secular resonance near $2 \mathrm{AU}$ (Williams and Faulkner, 1981) may effectively increase the inclinations of Apollo-Amor objects of asteroidal origin. Present Monte Carlo programs may not provide sufficiently reliable results for high inclination objects, but there is no fundamental reason this cannot be done. 


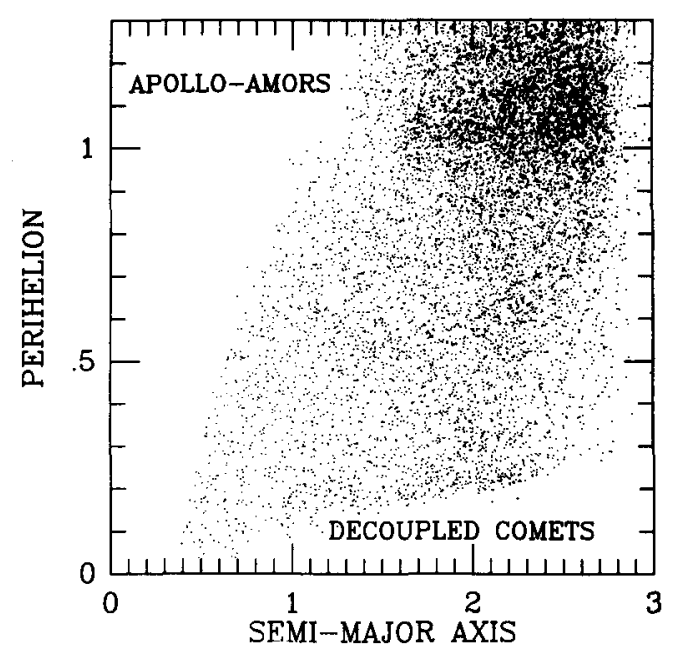

Figure 8. Steady-state distribution of Apollo-Amor orbits evolved from an initial cometary source. Although the semi-major axes of the initial orbits were clustered towards a $>2 \mathrm{AU}$, the orbits evolve to fill most of the permissible semi-major axis/perihelion space of Apollo-Amor objects.

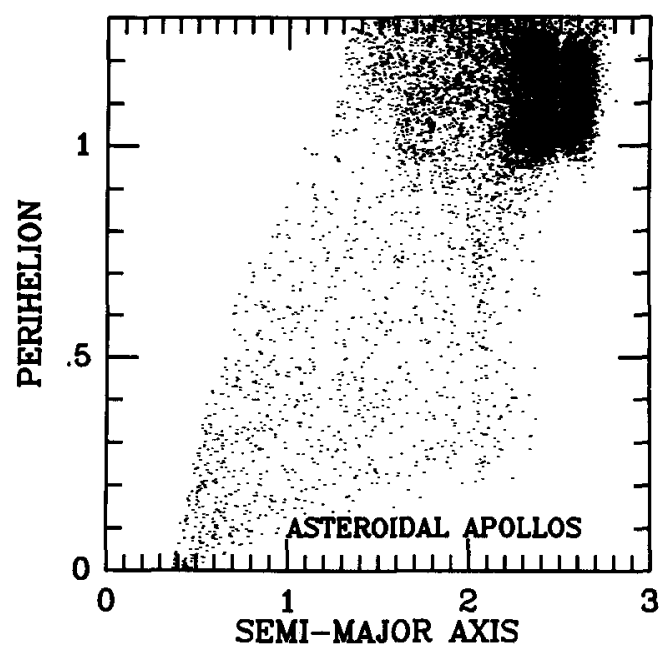

Figure 9. Steady-state distribution of Apollo-Amor objects evolved from a calculated asteroidal source (Wetherill, 1988). In the Apollo region (perihelia $<1 \mathrm{AU}$ ), the distribution is, in general, similar to that found for the cometary source (Figure 8), but includes fewer deep Earth-crossing Apollo objects with large semi-major axes. The principal difference between the cometary and asteroidal source is the large number of Amor objects, resulting from the marginal nature of initial Earth-crossing for an asteroidal source. 
The fact that most Apollo objects are not in orbits strongly correlated with a cometary origin should not be used to argue against comets and asteroids contributing a similar number of Apollo objects. In particular, it is likely to be misleading to consider, one by one, observed Apollo-Amor objects in evolved orbits, and classify them as likely to be of cometary or asteroidal origin on the basis of orbital evidence. The presence of a few bodies in orbits that are probably indicative of a cometary origin argues for a much larger population of cometary Apollos hidden among the general approximately equilibrium distribution. Classification based on albedo or reflectance spectra may prove to be more useful, but the effect of solar radiation on the surface properties of a comet after $\sim 10^{7}$ perihelion passages are at present unknown.

As mentioned in section 2, the orbital evolution from trans-Neptunian comets to Apollo objects and their ultimate loss from the Solar System is a continuous process. This continuity has been incorporated into calculations of the kind discussed in this paper. In the new results reported here, the orbital evolution of initial Neptune-crossing comets was followed until they were ejected from the Solar System or until their perihelion became less than $1 \mathrm{AU}$. Those ejected from the Solar System will not contribute to the ApolloAmor population; the other group may. At the next stage, the calculation of decoupling in section 4 , the orbits of a subset of this group, those with aphelia $<9$ AU and with inclinations less than $18^{\circ}$, were used as initial orbits. Those comets with aphelia $>9 \mathrm{AU}$, not belonging to Jupiter's family, could easily be included in the calculation, but they simply would not be decoupled. The high inclination objects were rejected because of the limitations of existing Monte Carlo techniques. This is not a fundamental limitation. Again, the orbital evolution of the objects used were followed until they were ejected, struck a planet, or became decoupled by definition when their aphelion became less than 4.35 AU. This definition is only apparently arbitrary. The orbital evolution of all the bodies were included: all those that first evolved into orbits with larger aphelia either evolved further inward or were ejected. All orbits that evolved into aphelia smaller than 4.35 AU, e.g., 4.0 AU, evolved from those with aphelia less than 4.35 AU. Finally, the calculation of the steady-state distribution shown in Figure 8 used as starting orbits the decoupled comet orbits shown in Figure 7.

\section{Concluding Remarks}

Much remains to be learned before the life history of comets from birth to banishment or death can be considered to be satisfactorily understood. To someone with a skeptical turn of mind, the evidence for a large fraction of the Apollo objects having originally been comets will not seem compelling. Nevertheless recent observational studies of comets, Apollo objects, and meteors; the existence of a number of bodies of asteroidal appearance in Jupiter-crossing orbits; physical modelling of the evolution of cometary nuclei; and the theoretical studies discussed here persuade the author that, more likely than not, comets are an important source of Earth-approaching bodies of asteroidal appearance.

Thus, old and rather worn-out comets may dominate the cometary population near Earth, be responsible for much or most of the impact cratering on the terrestrial planets and the Moon, contribute in an important way to the terrestrial flux of meteors and fireballs, and conceivably provide some small quantity of recoverable meteoritic material. 


\section{Acknowledgements}

I wish to thank Paul Weissman for teaching me much about real comets, and to Andrea Milani for helpful discussions regarding the orbital evolution of Apollo-Amor objects. I appreciate Janice Dunlap's work in preparing the manuscript. This work was supported by NASA grant NSG 7437.

\section{References}

Arnold, J. R. (1965) The origin of meteorites as small bodies. II. The model, III. General considerations, Astrophys. J. 141, 1536-1556.

Campins, H., A'Hearn, M. F., Schleicher, D. G., and Millis, R. L. (1988) The nucleus of comet P/Tempel 2, Bull. Amer. Astron. Soc. 20 (abstract).

Duncan, M., Quinn, T., and Tremaine, S. (1987) The formation and extent of the solar system comet cloud, Astrophys. J. 94, 1330-1338.

Duncan, M., Quinn, T., and Tremaine, S. D. (1988) The origin of short period comets, Astrophys. J. 328, L69-L73.

Everhart, E. (1972) The origin of short period comets, Astrophys. Lett. 10, 131-135.

Everhart, E. (1977) The evolution of comet orbits as perturbed by Uranus and Neptune, in A. H. Delsemme (ed.), Comets, Asteroids, Meteorites University of Toledo, Toledo, pp. 99-104.

Fernández, J. A. (1980) On the existence of a cometary belt beyond Neptune, Mon. Not. Roy. Astron. Soc. 192, 481-491.

Fernández, J. A., and W.-H. Ip (1983) On the time evolution of the cometary influx in the region of the terrestrial planets, Icarus 54, 377-387.

Fernández, J. A. (1985) Dynamical capture and physical decay of short-period comets, Icarus 64, 308-319.

Grieve, R. A. F., and Dence, M. R. (1979) The terrestrial cratering record II. The crater production rate, Icarus $38,230-242$.

Havnes, O. (1967) The effect of repeated close approaches to Jupiter on short-period comets, Icarus 12, 331-337.

Hills, J. G. (1981) Comet showers and the steady-state infall of comets from the Oort cloud, Astron. J. 86, 1730-1740.

Lecar, M., and Franklin, F. A. (1973) On the original distribution of the asteroids, I, Icarus 20, 422-436.

Marsden, B. G. (1985) Nongravitational forces on comets: The first fifteen years, in A. Carusi and G. B. Valsecchi (eds.), Dynamics of Comets: Their Origin and Evolution D. Reidel Publ. Co., Dordrecht, pp. 343-352.

Marsden, B. G. (1986) I.A.U. Catalogue of cometary orbits. Smithsonian Astrophysical Obs., Cambridge, Mass.

Milani, A., Hahn, G., Carpino, M., and Nobili, A. M. (1989) Dynamics of planet-crossing asteroids: Classes of orbital behaviour. Project Spaceguard, Icarus 78, 212-269. 
Öpik, E. J. (1951) Collision probabilities with the planets and the distribution of interplanetary matter, Proc. Roy. Irish Acad. 54A, 165-199.

Öpik, E. J. (1961) The survival of comets and cometary material, Astron. J. 66, 381-382.

Öpik, E. J. (1963) Survival of comet nuclei and the asteroids, Advan. Astron. Astrophys. 2, 219-262.

Scholl, H., and Froeschlé, C. H. (1986) The effects of the secular resonances $\nu_{16}$ and $\nu_{5}$ on asteroid orbits, Astron. Astrophys. 170, 134-144.

Sekanina, Z. (1969) Dynamical and evolutionary aspects of gradual deactivation and disintegration of short-period comets, Astron. J. 74, 1223-1234.

Sekanina, Z. (1971) A core-mantle model for cometary nuclei and asteroids of possible cometary origin, in T. Gehrels (ed.), Physical Studies of Minor Planets,NASA SP-267, pp. $423-428$.

Shoemaker, E. M. (1977) Astronomically observable crater-forming projectiles, in D. J. Roddy, R. O. Pepin and R. B. Merrill (eds.), Impact and Explosion Cratering Pergamon Press, New York, pp. 617-628.

Shoemaker, E. M., Williams, J. G., Helin, E. F., and Wolfe, R. F. (1979) Earth-crossing asteroids: orbital classes, collision rates with Earth, and origin, in T. Gehrels (ed.), Asteroids, Univ, of Arizona Press, Tucson, pp. 253-282.

Shoemaker, E. M., and Wolfe, R. F. (1984) Evolution of the Uranus-Neptune planetesimal swarm, Lunar Planet. Sci. XV, 780-781.

Stagg, C. R., and Bailey, M. E. (1989) Stochastic capture of short-period comets, Mon. Not. Roy. Astron. Soc.. In press.

Torbett, M. V. (1989) Chaotic motion in a comet disk beyond Neptune: The delivery of short-period comets, Preprint.

Weissman, P. R. (1980) Physical loss of long-period comets, Astron. Astrophys. 85, 191-196.

Weissman, P. R. (1986) The Oort cloud and the galaxy, in R. Smoluchowski, J. N. Bahcall and M. S. Matthews (eds.), The Galaxy and the Solar System, Univ. of Arizona Press, Tucson, pp. 204-237.

Weissman, P. R., A'Hearn, M. F., McFadden, L. A., and Rickman, H. (1989) Evolution of comets into asteroids, in press, in R. Binzel, T. Gehrels and M. S. Matthews (eds.), Asteroids II, Univ. of Arizona Press, Tucson.

Weissman, P. R. (1989) The cometary impactor flux at the Earth. Preprint of ms. submitted to Proceedings of the conference on global catastrophes in Earth history, Snowbird, Utah, October 1988. In press.

Wetherill, G. W. (1968a) Dynamical studies of asteroidal and cometary orbits and their relation to the origin of meteorites, in L. H. Ahrens (ed.), Origin and Distribution of the Elements, Pergamon, Oxford, pp. 423-443.

Wetherill, G. W. (1968b) Relationships between orbits and sources of chondritic meteorites, in P. Millman (ed.), Meteorite Research, D. Reidel Publ. Co., Dordrecht, pp. 573-589.

Wetherill, G. W. (1979) Steady-state population of Apollo-Amor objects, Icarus 37, 96112. 
Wetherill, G. W. (1985) Asteroidal source of ordinary chondrites, Meteoritics 20, 1-22.

Wetherill, G. W. (1987) Dynamic relationships between asteroids, meteorites, and ApolloAmor objects, Phil. Trans. Roy. Soc. of London A323, 323-337.

Wetherill, G. W. (1988) Where do the Apollo objects come from? Icarus 76, 1-18.

Wetherill, G. W. (1989) Cratering of the terrestrial planets by Apollo objects, Meteoritics 24, 15-22.

Wetherill, G. W., and Williams, J. G. (1968) Evaluation of the Apollo asteroids as sources of stone meteorites, J. Geophys. Res. 73, 635-648.

Wetherill, G. W., and Williams, J. G. (1979) Origin of Differentiated Meteorites, in L. H. Ahrens (ed.), Origin and Distribution of the Elements Pergamon, Oxford, pp. 19-31.

Whipple, F. L. (1950) A comet model I. The acceleration of comet Encke, Astrophys. J. 111, 375-394.

Whipple, F. L. (1951) A comet model II. Physical relations for comets and meteors, Astrophys. J. 113, 464-474.

Williams, J. G. (1973) Meteorites from the asteroid belt? (abstract), Eos 54, 233.

Williams, J. G. and Faulkner, J. (1981) The positions of secular resonant surfaces, Icarus 46, 390-399.

Wisdom, J. (1983) Chaotic behavior and the origin of the $3 / 1$ Kirkwood gap, Icarus 56, 51-74.

Wisdom, J. (1985) Meteorites may follow a chaotic route to Earth, Nature 315, 731-733.

Yeomans, D. K. (1988) A new look at cometary nongravitational forces, Bull. Am. Astron. Soc. 20, 841-842. 